\title{
Diabetes tipo 2 en niños. Reporte de caso.
}

\author{
Type 2 diabetes in children. A case report.
}

Miguel Pinto ${ }^{1,3}$, Segundo Seclén ${ }^{1,3}$, Emilio Cabello ${ }^{2,3}$.

\section{RESUMEN}

Se reporta el caso de una niña de 14 años con antecedentes de obesidad, dislipidemia y diabetes que es referida a nuestro servicio por polidipsia, poliuria y sobrepeso. La paciente tenía el antecedente familiar de ambos padres con diagnóstico de diabetes tipo 2. El examen físico mostró obesidad central y acantosis nigricans severa en los pliegues cutáneos. Los análisis de laboratorio mostraron hiperglicemia, hiperinsulinemia y péptido C normal. Los autoanticuerpos anti-GAD y anti-ICA fueron negativos. El tratamiento con metformina fue continuado; y la dieta y actividad física fueron reforzadas. Actualmente, los niveles de hemoglobina glicosilada y glicemia en ayunas han mejorado. El incremento en la obesidad infantil ha resultado en un marcado aumento en la incidencia de síndrome metabólico y diabetes tipo 2 en la población infantil.(Rev Med Hered 2010;21:103-106).

PALABRAS CLAVE: Diabetes tipo 2, obesidad, acantosis nigricans, resistencia a la insulina, síndrome metabólico

\section{SUMMARY}

We report a 14-year-old girl with history of diabetes, obesity and, dyslipidemia. The patient was referred to our service because of polydipsia, polyuria and, overweight. Both parents were patients with type 2 diabetes. Physical examination showed abdominal obesity and severe acanthosis nigricans in skin folds. Laboratory results showed hyperglycemia, hyperinsulinemia, normal C-peptide and, the autoantibodies against islet antigens were negative. Treatment with metformin was continued. Diet and physical activity were reinforced. Currently, hemoglobin A1c and fasting plasma glucose have been improved.The rise in childhood obesity has resulted in a marked increase in the incidence of metabolic syndrome and type 2 diabetes in pediatric population.(Rev Med Hered 2010;21:103106).

KEY WORDS: Type 2 diabetes, obesity, insulin resistance, children, acanthosis nigricans, metabolic syndrome.

Servicio de Endocrinología, Hospital Nacional Cayetano Heredia. Lima, Perú.

Unidad de Endocrinología Pediátrica, Hospital Nacional Cayetano Heredia. Lima, Perú.

Facultad de Medicina Alberto Hurtado, Universidad Peruana Cayetano Heredia. Lima, Perú. 


\section{INTRODUCCIÓN}

La diabetes tipo 2 (DM2) ha sido tradicionalmente considerada una enfermedad de adultos (1); sin embargo, a medida que la prevalencia de obesidad aumenta en la población infantil, los casos de DM2 en niños y adolescentes se irán incrementando. Este problema afecta de manera especial a niños de grupos étnicos de riesgo, como los hispanos (2,3). A mediados de los años 90, los casos de DM2 en niños pasaron de ser reportes aislados a representar el $16 \%$ de todos los casos nuevos de diabetes en zonas urbanas (4). Actualmente, la incidencia de casos de DM2 en niños de grupos étnicos de riesgo se aproxima al 50\% de todos los nuevos casos de diabetes en niños y adolescentes de los Estados Unidos (5).

Los factores asociados con la aparición de DM2 en esta población son: genéticos, obesidad, sedentarismo, resistencia a la insulina, ambiente intrauterino, historia familiar y bajo status socioeconómico $(6,7,8)$. La resistencia a la insulina asociada a la obesidad va ha provocar un deterioro progresivo de la función de la célula beta del páncreas, produciendo diversos grados de hiperglicemia hasta la aparición de diabetes $(5,9)$. La resistencia a la insulina asociada con la pubertad podría acelerar este deterioro (10).

En este reporte, describimos el caso de una niña con antecedentes de obesidad y dislipidemia, quien desarrolla diabetes tipo 2 .

Nuestro objetivo, es llamar la atención acerca de la aparición de casos de diabetes tipo 2 en niños de nuestro medio, asociados a la epidemia de obesidad que se presenta en nuestro país. Estos niños van a presentar a temprana edad las clásicas complicaciones de la diabetes, es decir, insuficiencia renal, ceguera y enfermedades cardiovasculares.

\section{Caso clínico}

Niña de 14 años de edad que fue referida a nuestro servicio por presentar polidipsia, poliuria y sobrepeso. La paciente tenía el diagnóstico de sobrepeso desde los 4 años de edad y dislipidemia desde los 8 años. Inicialmente, recibió tratamiento con dieta y ejercicio de manera irregular. A los 11 años fue diagnosticada de diabetes por vulvovaginitis, poliuria, polidipsia y glicemia en ayunas de $282 \mathrm{mg} / \mathrm{dl}$. A partir de esa fecha, recibió de manera irregular metformina $850 \mathrm{mg}$ dos veces al día. La dieta y ejercicio continuaban siendo irregulares. Además, tenía el antecedente familiar de madre, padre y abuela materna con DM2.

El examen físico mostró obesidad abdominal (IMC 33.6) y acantosis nigricans severa a nivel del cuello y otros pliegues cutáneos. Su desarrollo según la escala de Tanner era de 4.

Los exámenes auxiliares en ayunas mostraron: glicemia de $269 \mathrm{mg} / \mathrm{dl}$, insulina de $29 \mathrm{mIU} / \mathrm{mL}$ (VN: 627), péptido C de 4,6 ng/dl (VN: 1,1-5,0), hemoglobina glicosilada A1c (HbA1c) de 9,2\% (VN: <6,5), colesterol total de $154 \mathrm{mg} / \mathrm{dl}$ (VN: <170), HDL colesterol de 38 mg/dl (VN: > 34), LDL colesterol de 94 mg/dl (VN: < 100) y triglicéridos de $109 \mathrm{mg} / \mathrm{dl}$ (VN: < 200). Los autoanticuerpos anti-GAD y anti-ICA fueron negativos.

El tratamiento con metformina $850 \mathrm{mg}$ dos veces al día fue continuado, además se intensificó la terapia con dieta y actividad física regular. Después de 14 semanas, la glicemia en ayunas fue de $196 \mathrm{mg} / \mathrm{dl}$ y la HbA1c de 8,9\%, respectivamente.

Posteriormente, la dosis de metformina fue incrementada a $1000 \mathrm{mg}$ dos veces al día. Después de 28 semanas de tratamiento, la glicemia en ayunas disminuyó a $162 \mathrm{mg} / \mathrm{dl}$ y la HbA1c fue de 7,9\%. Actualmente, la paciente continúa tratamiento con metformina $1000 \mathrm{mg}$ dos veces al día, dieta y ejercicios estrictos. Las evaluaciones anuales de fondo de ojo, microalbuminuria y pies fueron normales.

\section{DISCUSIÓN}

Hace 15 años, los casos de DM2 en niños representaban menos del 3\% de todos los casos nuevos de diabetes en niños y adolescentes, sin embargo en nuestros días, representan el $45 \%$ de todos los casos de diabetes. Estos pacientes generalmente son obesos, y por lo tanto, con riesgo a desarrollar otras enfermedades como hipertensión arterial, dislipidemia, hígado graso no alcohólico y síndrome metabólico; las cuales están asociadas con un mayor riesgo cardiovascular (11).

La obesidad es el factor crítico y potencialmente modificable asociado a la aparición de resistencia a la insulina y DM2 $(4,6)$. La resistencia a la insulina es una característica temprana de los niños con DM2. Inicialmente, se produce una hiperinsulinemia compensatoria, sin embargo, con el tiempo se desarrolla 
una falla en la secreción de insulina por la célula beta del páncreas, resultando en la expresión clínica de DM2 (8).

La historia familiar, es otro factor asociado a DM2 en este grupo de sujetos $(6,12)$, de los cuales, entre el $45-80 \%$ tienen al menos un padre con diabetes, y entre el $74-100 \%$ tienen un familiar en primer o segundo grado con diabetes.

El ambiente intrauterino, a través del peso al nacer y la hiperglicemia materna, puede afectar el desarrollo de DM2 en la niñez $(8,13)$. El riesgo de DM2 es mayor entre aquellos con peso al nacer menor a 2500 gramos o mayor a 4000 gramos. Por otro lado, los hijos de mujeres que desarrollan diabetes durante la gestación, tienen más riesgo de ser obesos en la infancia y de desarrollar diabetes en su segunda década de vida.

Otros factores asociados son: inactividad física (mayor riesgo de sobrepeso y obesidad), acantosis nigricans (marcador de resistencia a la insulina, presente en $60-90 \%$ de los niños con DM2), sexo femenino (las niñas tienen 1.7 veces el riesgo de desarrollar DM2), síndrome de ovario poliquístico (asociado a resistencia a la insulina e hiperinsulinemia compensatoria, donde $30 \%$ de las adolescentes afectadas tienen intolerancia a la glucosa y $4 \%$ el diagnóstico de DM2) y status socio-económico (mayor riesgo asociado a menor status socio-económico) $(6,8,12)$.

Respecto al tratamiento, la mayoría de agentes farmacológicos no han sido aprobados para ser usados en niños, y lo mismo se aplica para los fármacos usados para el tratamiento de la hipertensión y dislipidemia.

Los objetivos del tratamiento de DM2 en niños y adolescentes son: bienestar físico y mental, control glicémico adecuado y prevención de complicaciones micro y macrovasculares (8). Los cambios en estilo de vida (dieta y actividad física) son difíciles de mantener en la población infantil. En general, menos del $10 \%$ de los adolescentes alcanzan las metas del control metabólico adecuado (HbA1c menor a 7\%) solamente con dieta y ejercicio (9) y estos cambios, deben incluir al entorno familiar (5). La metformina es el único agente oral aprobado para el tratamiento de DM2 en niños y adolescentes. La metformina reduce la producción hepática de glucosa e incrementa la sensibilidad a la insulina (13). Un estudio demostró que metformina a dosis de $2000 \mathrm{mg}$ por día, disminuyó la glicemia en ayunas en 42,9 mg/dl y la HbA1c en desde
$8,2 \%$ a $7,2 \%$. En general, el $84 \%$ de los participantes en este estudio alcanzaron la meta de glicemia en ayunas menor a $126 \mathrm{mg} / \mathrm{dl}$ o HbA1c menor a 7\% (14).

De manera similar que los adultos, los niños con DM2 tienen una mayor prevalencia de comorbilidades como la hipertensión arterial y la dislipidemia (11). Un estudio demostró que 49\% y 11\% de jóvenes con DM2 tienen presión arterial sistólica y diastólica respectivamente, por encima del 95\% percetil para la edad, sexo y talla al momento de presentación de la enfermedad (15). La prevalencia de dislipidemia al diagnóstico en niños y adolescentes con DM2 varía entre $18-61 \%$, y menos del $1 \%$ reciben medicación hipolipemiante (11).

En conclusión, la epidemia de obesidad en la población infantil esta produciendo un aumento en la incidencia de casos de diabetes tipo 2 en niños y adolescentes. Estos pacientes pueden tener asociados otros factores de riesgo como hipertensión arterial y dislipidemia. El tratamiento de estos niños se basa en estilos de vida saludables y el uso de metformina. A largo plazo, tienen un riesgo incrementado de complicaciones como nefropatía, retinopatía y enfermedad cardiovascular.

\section{Correspondencia:}

Miguel Pinto Valdivia

Servicio de Endocrinología, Hospital Nacional Cayetano Heredia (Lima, Perú)

Avenida Honorio Delgado 262, Lima 31, Perú

Teléfono: +511 4814496

Fax: +511 3812631

Correo electrònico: miguelpinto72@yahoo.com

\section{REFERENCIAS BIBLIOGRAFICAS}

1. Shaw J. Epidemiology of childhood type 2 diabetes and obesity. Pediatric Diabetes 2007; 8 (9): 7-15.

2. Cali AM, Caprio S. Prediabetes and type 2 diabetes in youth: an emerging epidemic disease? Curr Opin Endocrinol Diabetes Obes 2008; 15: 123-127.

3. Pohl JH, Greer JA, Hasan KS. Type 2 Diabetes Mellitus in Children. Endocr Pract 1998; 4: 413-416.

4. Kaufman FR. Obesity and type 2 diabetes in children and youth. Curr Opin Endocrinol Diabetes 2006; 13: 332-337.

5. Weigensberg MJ, Goran MI. Type 2 diabetes in children and adolescents. Lancet 2009; 373: 1743-1744.

6. Dabelea D, Pettitt DJ, Jones KL, Arslanian SA. Type 2 diabetes mellitus in minority children and adolescents. 
An emerging problem. Endocrinol Metab Clin North Am 1999; 28: 709-729.

7. American Diabetes Association. Type 2 diabetes in children and adolescents. Diabetes Care 2000; 23: 381-389.

8. Alberti G, Zimmet P, Shaw J, Bloomgarden Z, Kaufman F, Silink M. Type 2 diabetes in the young: The evolving epidemic. The International Diabetes Federation Consensus Workshop. Diabetes Care 2004; 27: 1798-1811.

9. Kaufman FR. Type 2 diabetes in children and youth. Rev Endocr Metab Disord 2003; 4: 33-42.

10. Goran MI, Gower BA. Longitudinal study on pubertal insulin resistance. Diabetes 2001; 50: 24442450.

11. Pinhas-Hamiel O, Zeitler P. Acute and chronic complications of type 2 diabetes mellitus in children and adolescents. Lancet 2007; 369: 1823-1831.

12. Singh R, Shaw J, Zimmet P. Epidemiology of childhood type 2 diabetes in the developing world. Pediatric diabetes 2004; 5: 154-168.

13. Freemark M. Pharmacologic approaches to the prevention of type 2 diabetes in high risk pediatric patients. J Clin Endocrinol Metab 2003; 88: 3-13.

14. Jones KL, Arslanian S, Peterokova VA, Park JS, Tomlinson MJ. Effect of metformin in pediatric patients with type 2 diabetes. Diabetes Care 2002; 25: 89-94.

15. Upchurch SL, Brosnan CA, Meininger JC, et al. Characteristics of 98 children and adolescents diagnosed with type 2 diabetes by their health care provider at initial presentation. Diabetes Care 2003; 26: 2209.

Recibido: 26/09/09

Aceptado para publicación:22/04/10 